\title{
EagI channels as potential early-stage biomarkers of hepatocellular carcinoma
}

This article was published in the following Dove Press journal:

Biologics:Targets and Therapy

20 September 2016

Number of times this article has been viewed

\author{
María de Guadalupe \\ Chávez-López' \\ Violeta Zúñiga-García' \\ Julio Isael Pérez-Carreón ${ }^{2}$ \\ Arturo Avalos-Fuentes ${ }^{3}$ \\ Yesenia Escobar ${ }^{4}$ \\ Javier Camacho' \\ 'Department of Pharmacology, \\ Centro de Investigación y de \\ Estudios Avanzados del Instituto \\ Politécnico Nacional, ${ }^{2}$ Instituto \\ Nacional de Medicina Genómica, \\ ${ }^{3}$ Department of Physiology, Biophysics \\ and Neuroscience, Centro de \\ Investigación y de Estudios Avanzados \\ del Instituto Politécnico Nacional, \\ ${ }^{4}$ Centro de Investigación Clínica \\ Acelerada Sc, Mexico City, Mexico
}

Correspondence: Javier Camacho Department of Pharmacology, Centro de Investigación y de

Estudios Avanzados del Instituto Politécnico Nacional, Avenida Instituto Politécnico Nacional 2508, CP 07360 ,

Mexico City, Mexico

Tel +525557473800 ext 5416

Fax +525553430106

Email fcamacho@cinvestav.mx

\begin{abstract}
Hepatocellular carcinoma (HCC) is a major cause of cancer death worldwide. HCC is usually asymptomatic at potential curative stages, and it has very poor prognosis if detected later. Thus, the identification of early biomarkers and novel therapies is essential to improve HCC patient survival. Ion channels have been proposed as potential tumor markers and therapeutic targets for several cancers including HCC. Especially, the ether à-go-go-1 (Eag1) voltage-gated potassium channel has been suggested as an early marker for HCC. Eag1 is overexpressed during HCC development from the cirrhotic and the preneoplastic lesions preceding $\mathrm{HCC}$ in a rat model. The channel is also overexpressed in human HCC. Astemizole has gained great interest as a potential anticancer drug because it targets several proteins involved in cancer including Eag1. Actually, in vivo studies have shown that astemizole may have clinical utility for HCC prevention and treatment. Here, we will review first some general aspects of HCC including the current biomarkers and therapies, and then we will focus on Eag1 channels as promising tools in the early diagnosis of HCC.
\end{abstract}

Keywords: ion channels, Eag1, hepatocellular carcinoma, astemizole, diethylnitrosamine

\section{Introduction}

Primary liver cancer is a major health problem, ${ }^{1}$ representing the second leading cause of cancer-related deaths in the world. ${ }^{2}$ Hepatocellular carcinoma (HCC) accounts for up to $90 \%$ of primary liver cancers. ${ }^{1}$ Other liver cancer types include childhood hepatoblastoma, adult cholangiocarcinoma (originating from the intrahepatic biliary ducts), and angiosarcoma (from the intrahepatic blood vessels). ${ }^{3}$

$\mathrm{HCC}$ affects men three times more frequently than women worldwide. It is the fifth most frequently diagnosed cancer in adult men, and the seventh most commonly diagnosed cancer in adult women. ${ }^{4}$ Interestingly, significant differences have been noted between the races. Asians are affected two times more than blacks, and Hispanics are affected two times more than whites. The ethnic variability reflects the contribution of specific causal factor differences among populations. ${ }^{4}$ Because of its poor prognosis, it is compulsory to find novel early markers of the disease as well as new therapeutic approaches for HCC prevention and treatment.

\section{HCC pathophysiology}

Liver cirrhosis is the strongest HCC predisposing factor. Actually, 80\% of HCC cases formerly developed cirrhosis. Other well-defined risk factors comprise viral infections (chronic hepatitis B and C), toxics (alcohol and aflatoxin B1), and altered 
Table I Main HCC risk factors

\begin{tabular}{ll}
\hline Risk factor & Description \\
\hline Liver cirrhosis & This is the most important clinical risk factor for HCC. The transition from chronic liver disease to cirrhosis involves \\
& inflammation and activation of hepatic stellate cells with ensuing fibrogenesis and angiogenesis. As many hepatocytes are
\end{tabular}
lost, the liver loses the ability to metabolize bilirubin (which can result in an increased serum bilirubin and transaminase level). Liver cirrhosis is characterized by diffuse nodular regeneration surrounded by dense fibrotic septa with subsequent parenchymal extinction and liver structure collapse. ${ }^{58,59}$

HBV infection The hepatitis B virus is a DNA virus belonging to the Hepadnaviridae family. Many evidences support that HBV DNA is frequently integrated into the chromosomal DNA of hepatocytes in most HBV-infected patients. The mechanisms of carcinogenesis in HBV infection have been extensively studied, and a major factor is chronic necroinflammation with subsequent fibrosis and hepatocyte proliferation. However, $\mathrm{HCC}$ may occur in $\mathrm{HBsAg}$ carriers without cirrhosis. Therefore, viral factors are likely involved in HBV-related hepatocarcinogenesis. For instance, the HBx protein promotes cell cycle progression, inactivates negative growth regulators, and binds to and inhibits the expression of p53 and other tumor suppressor genes and senescence-related factors. ${ }^{5,6,60-62}$

HCV infection $\quad \mathrm{HCV}$ is an enveloped, single-stranded, positive-sense RNA virus. Up to $80 \%$ of HCV-infected individuals fail to eliminate the virus acutely and progress to chronic HCV infection. Continuous inflammation and hepatocyte regeneration in the setting of chronic hepatitis and subsequent progression to cirrhosis are thought to lead to chromosomal damage and possibly to initiate hepatic carcinogenesis. HCV also induces steatosis; oxidative stress causes steatohepatitis and these pathways lead to liver injury or HCC in chronic HCV infection. ${ }^{6,63,64}$

Aflatoxin $\left(\mathrm{AFB}_{1}\right)$ This is a difuranocoumarin-derivative mycotoxin from Aspergillus flavus and Aspergillus parasiticus. It is produced under certain climatic factors and storage techniques favoring the fungus growth and contaminating foods such as maize, groundnuts, rice, and sorghum. IARC ranked $A F B_{1}$ as the most potent experimental hepatocarcinogen, and it is causally related to the development of HCC in humans.

Hepatitis $B$ virus infection may directly or indirectly sensitize hepatocytes to the carcinogenic effects of $A F B_{1}$, raising the possibility of a synergistic hepatocarcinogenic interaction between these two agents, although the precise mechanism is still not well understood..$^{6,65}$

Alcohol Alcohol use has definitely been recognized as a cause of HCC. This may be well related to the development of HCC due to direct (genotoxic) and indirect factors (cirrhosis development). ADH metabolizes ethanol to acetaldehyde; this metabolite is not only extremely toxic but also carcinogenic. Alcohol-related liver cirrhosis is most likely the main risk factor for HCC in groups of people with low rates of hepatitis B or C viral infection, such as the US and Northern Europe. Some reports mention that $25-80 \mathrm{~g} / \mathrm{d}$ of alcohol use for 10 years or more for men, and $12-20 \mathrm{~g} / \mathrm{d}$ for women, increases the risk of developing cirrhosis. ${ }^{1,66}$

DM This metabolic disorder may predispose the liver to relative insulin resistance due to inadequate insulin secretion or receptor insensitivity to endogenous insulin. Several evidences showed that insulin resistance and DM induce the progression of NAFLD, including its most severe form, NASH, which has been identified as a cause of cirrhosis and HCC.,67

NAFLD NAFLD defines liver abnormalities ranging from simple steatosis (abnormal hepatic fat accumulation) or nonalcoholic fatty liver to NASH with or without cirrhosis development. Importantly, it is also closely linked to obesity and metabolic syndromes.

Although there is significant progress in understanding carcinogenesis, the exact mechanism of HCC development from NAFLD has not yet been fully elucidated. However, a recent report suggests that the antiapoptotic protein survivin is differently expressed in NASH-HCC-related tissues compared with HCV-HCC-related samples. Besides, another study identified that mTOR was differently expressed in NAFLD-related cirrhosis compared with other causes of cirrhosis. ${ }^{68,69}$

HH $\quad \mathrm{HH}$ is an inherited (genetic) disorder causing the body to absorb too much iron from the diet. The main storage sites of iron are the hepatocytes, and this metal is essential for their normal functioning, although iron is ubiquitous in human cells. The hepatotoxic and hepatocarcinogenic potential of excessive iron $(>5 \mathrm{~g})$ is due to its ability to generate ROS intermediates and oxidative stress (by the Fenton reaction). This stress damages DNA, lipids, and proteins, resulting in necrosis and apoptosis of hepatocytes. Patients with $\mathrm{HH}$ have an estimated 240 -fold increased relative risk of developing HCC., 10

Tobacco use Tobacco smoking has been suggested as a significant HCC risk factor. Nicotine, the main component of cigarette smoke, upregulates CYP2EI activity in the liver of rodents and humans. CYP2EI induction is associated with ROS generation and lipid peroxidation, which may be some mechanisms whereby tobacco smoke contributes to HCC. ${ }^{71}$

Obesity

Obesity has been associated with HCC because lipid accumulation within hepatocytes leads to a chronic low-grade inflammation involving the release of proinflammatory cytokines and inhibition of anti-inflammatory cytokines, leading to hyperinsulinemia. Increased levels of IGF-I have important proliferative and antiapoptotic effects. This factor also promotes angiogenesis via increased vascular endothelial growth factor production, which in turn leads to proliferation of cancer cells. ${ }^{72}$

OCs OCs are combinations of estrogens and progestogens. Synthetic estrogens such as ethinyl estradiol and mestranol produce malignant liver tumors in rodents, probably by acting as promoting agents. Additionally, progestogens (norethisterone and norethynodrel) cause benign liver neoplasia in animals. OCs may cause liver cancer by mitogenesis because increased proliferation rates may enhance the rate of spontaneous mutations due to DNA polymerase errors. ${ }^{1,54}$

Abbreviations: $\mathrm{ADH}$, alcohol dehydrogenase; DM, diabetes mellitus; $\mathrm{HBsAg}$, hepatitis B surface antigen; HBV, hepatitis B virus; $\mathrm{HCC}$, hepatocellular carcinoma; $\mathrm{HCV}$, hepatitis C virus; HH, hereditary hemochromatosis; IARC, International Agency for Research on Cancer; IGF-I, insulin growth factor-I; mTOR, mechanistic target of rapamycin; NAFLD, nonalcoholic fatty liver disease; NASH, nonalcoholic steatohepatitis; OCs, oral contraceptives; ROS, reactive oxygen species. 
metabolic conditions (diabetes, nonalcoholic fatty liver disease, hereditary hemochromatosis). ${ }^{5-7}$ Recently, tobacco and obesity have also been proposed as potential risk factors for the development of $\mathrm{HCC}^{8}$ The major risk factors for $\mathrm{HCC}$ are described in Table 1.

Hepatocarcinogenesis is a multistep process in which a number of genetic alterations accumulate in the cells. After hepatic injury due to major predisposing risk factors for HCC, necrosis arises followed by hepatocyte proliferation. This continuous process of destructive-regenerative cycles in the hepatocyte promotes chronic liver injury and progressive liver fibrosis resulting in cirrhosis. If the process continues, the next step is the progressive malignant transformation of cirrhotic nodules and premalignant lesions, which will finally lead to $\mathrm{HCC}^{9,10}$ (Figure 1).

Because most HCC cases are associated with chronic viral hepatitis, prevention of virus infection should lead to the prevention of HCC. ${ }^{11-13}$ Some preventive strategies already used include vaccination (the universal vaccination was introduced in all newborns and high-risk groups as routine immunization ${ }^{13-15}$ ), antiviral treatment (it has been shown that antiviral treatment of chronic hepatitis B virus and hepatitis $\mathrm{C}$ virus infections may reduce the risk of $\mathrm{HCC}^{13,16,17}$ ), and periodical surveillance in patients at risk of developing a disease (Figure 2). ${ }^{11,13,18}$

\section{HCC diagnosis and treatment Diagnosis}

$\mathrm{HCC}$ is frequently diagnosed at an asymptomatic stage while the patients are being evaluated for liver transplantation or as part of routine screening in cirrhotic patients. The classic clinical features of HCC include right upper quadrant pain, weight loss, and worsening of liver function. Patients with chronic liver diseases belong to a high-risk group for HCC and a follow-up based on imaging and tumor marker levels ${ }^{11,19}$ should be regularly made for early diagnosis.

\section{Current and emerging potential HCC biomarkers}

Alfa-fetoprotein (AFP) is commonly used as an HCC marker; serum AFP levels may be helpful in the diagnosis and management of HCC. AFP is higher than $20 \mathrm{ng} / \mathrm{mL}$ in more than $70 \%$ of HCC patients. However, AFP levels from 10 to $500 \mathrm{ng} / \mathrm{mL}$ and even up to $1,000 \mathrm{ng} / \mathrm{mL}$ may be found in patients with other liver diseases who do not have HCC. AFP is useful mainly in monitoring the response to treatment

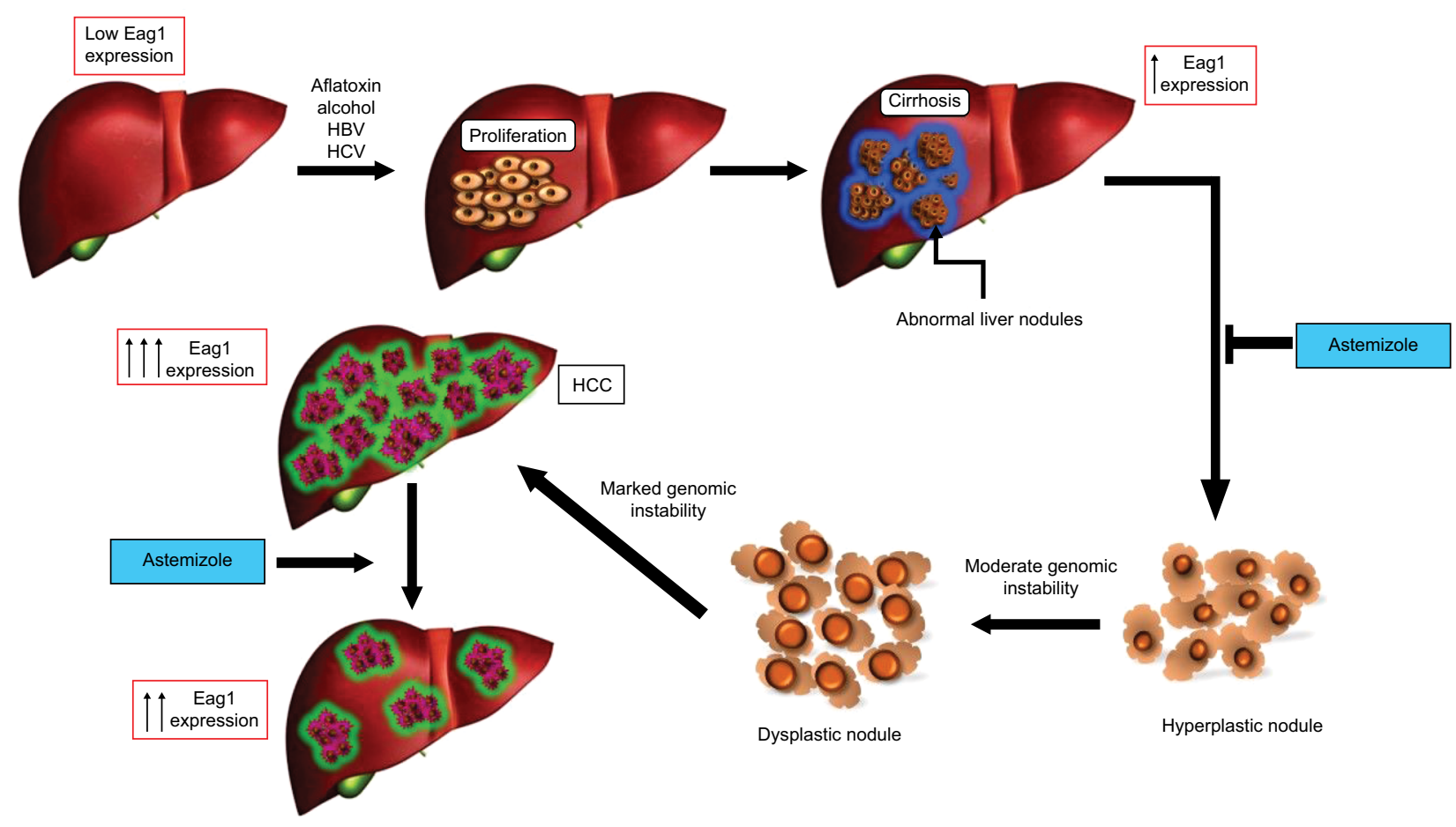

Figure I Eagl expression in the progression of HCC.

Notes: The principal risk factors for the development of HCC induce serious liver damage, causing necrosis and proliferation in the hepatocyte. This phase is known as chronic liver disease and may continue to liver cirrhosis. Cirrhosis is characterized by the presence of fibrosis; during this process, the connective tissue separates the liver into multiple regeneration nodules, that is, the fibrosis surrounds the nodules completely. The hyperplastic nodules evolve to dysplastic ones and ultimately to HCC. The use of astemizole in the cirrhosis stage may prevent the development of HCC and even produce HCC regression. Eagl channels have been shown to be expressed at early stages of HCC development. ${ }^{32}$

Abbreviations: Eagl, ether à-go-go-I; HCC, hepatocellular carcinoma. 


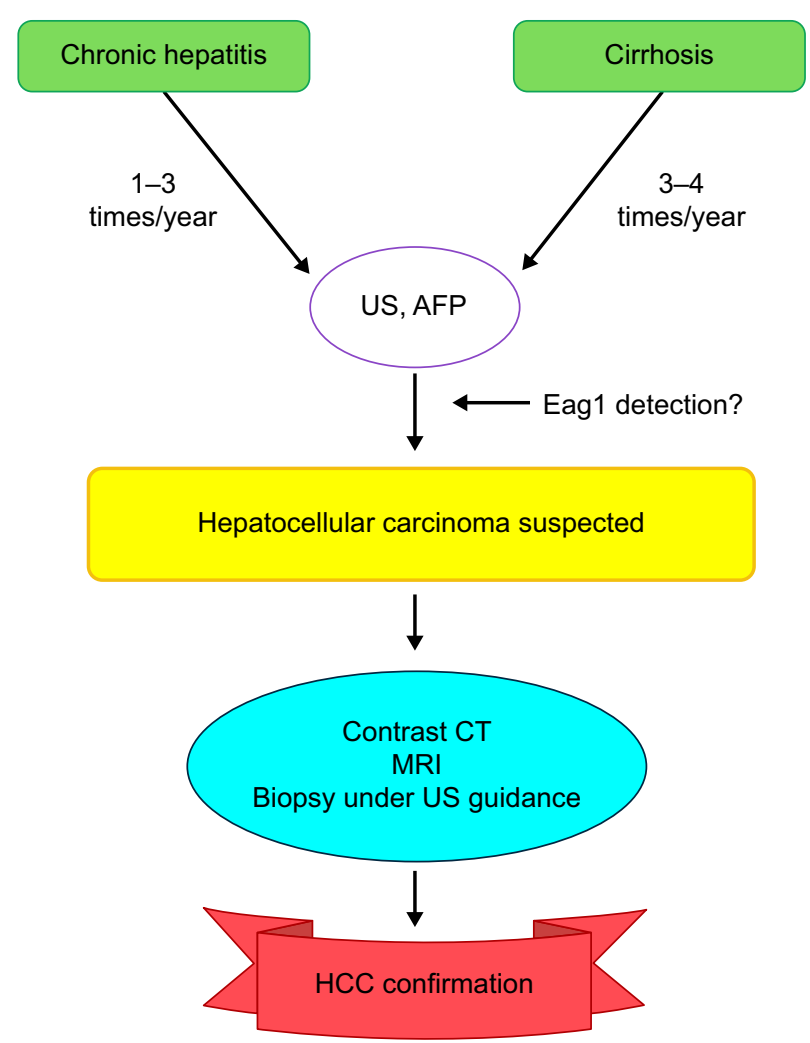

Figure 2 Follow-up for patients at risk of HCC.

Notes: The early screening should help to detect HCC when treatable. This screening should be performed, especially in patients with liver diseases at risk of developing HCC, and Eagl detection may be also included in this screening.

Abbreviations: AFP, alfa-fetoprotein; CT, computed tomography; Eagl, ether à-go-go-I; HCC, hepatocellular carcinoma; MRI, magnetic resonance imaging; US, ultrasound.

and detecting recurrence after treatment of $\mathrm{HCC}$, if the AFP levels were elevated before treatment. ${ }^{11,12,19,20}$

Despite that an ideal biomarker should have high sensitivity and specificity ${ }^{21}$ and be detectable in any of different samples such as blood, urine, or tissues ${ }^{22}$ the coexistence of inflammation and cirrhosis in HCC makes the early diagnosis and prognostic assessment much more difficult. This complication highlights the need to identify valuable biomarkers for the diagnosis and treatment of HCC.22

Recent advances in genomics and proteomics have facilitated the identification of many biomarkers, including new HCC molecular markers. However, only a few biomarkers are acceptable for clinical utility because the rest have low predictive accuracy and/or high cost. ${ }^{23,24}$ The most relevant biomarkers in liver cancer are described in Table 2.

Most of these markers have been studied in a retrospective manner, and only a few prospective trials have evaluated their clinical significance. However, as HCC is a complex disease with multiple underlying pathogenic mechanisms caused by a variety of risk factors, it is difficult to characterize
HCC with a single biomarker. In addition, most of the used biomarkers are associated with liver damage and not HCC itself. Therefore, novel HCC markers and diagnostic approaches are needed. The combination of biomarkers in a detection kit may be more valuable for the diagnosis and prognosis of HCC. It is also important to identify noninvasive and cost-effective biomarkers for early diagnosis.

\section{Imaging}

Ultrasound (US) studies still play an important role in detecting HCC because US is able to detect small liver lesions and has relatively high sensitivity and specificity. Its use in diagnosis is being replaced by computed tomography and magnetic resonance imaging, especially for those patients whose liver cannot be fully imaged by US because the tumors are very small or whose echograms do not show lesions clearly. ${ }^{11,12,19}$

\section{Biopsy}

If a tumor is detected by imaging, a biopsy can be obtained typically with a needle under US radiologic guidance. The material obtained by fine-needle aspiration is evaluated to determine HCC staging. ${ }^{11,12,19}$ Because HCC patients are diagnosed at advanced stages of the disease, it is advisable to screen patients who are at risk of developing the disease like those with chronic hepatitis and cirrhosis for early diagnosis (Figure 2). The most common classification of HCC depends on its stage (from early to terminal) and its differentiation grade (well, moderately, or poorly differentiated). ${ }^{13}$

\section{Treatment}

HCC treatment depends on the tumor stage, patient performance status, and liver function and requires a multidisciplinary approach. The treatment strategies of HCC are summarized in Table 3.

Unfortunately, HCC is among the most chemoresistant tumors. Chemotherapy with cytotoxic agents, such as doxorubicin, gemcitabine, cisplatin, and 5-fluorouracil, or combined regimen for palliative care is associated with low response rates. Interestingly, targeted chemotherapy with multikinase inhibitors (Table 4) seems to be an attractive alternative to conventional systemic chemotherapy.

Electrochemotherapy is a treatment used in cutaneous and subcutaneous tumors with positive results. Its use in deep tumors has been studied. The study showed favorable results in the treatment of liver metastases. Therefore this 
Table 2 Principal and potential HCC biomarkers

AFP

AFP is a fetal component protein produced in the yolk sac and liver of the developing fetus, it has been considered as the most useful serum protein for the diagnosis of patients at risk of HCC.

High AFP serum levels have been found in $60 \%-70 \%$ of patients with $\mathrm{HCC}$. Nevertheless, it is not HCC specific since elevated AFP levels are observed in liver cirrhosis and other tumors, including lung, biliary, gastric, and pancreatic cancer. AFP levels $<20 \mathrm{ng} / \mathrm{mL}$ are considered normal, while high levels of AFP ( $>400 \mathrm{ng} / \mathrm{mL}$ ) are strongly predictive for HCC. This diagnostic test has sensitivity values of $41 \%-65 \%$ to diagnose $\mathrm{HCC}$, but low specificity. ${ }^{24,73}$

DKKI DKKI is a serum protein marker for $\mathrm{HCC}$ and plays an important role in HCC progression through the promotion of cytoplasmic/nuclear accumulation of beta-catenin in $\mathrm{HCC}$ cells via the Wnt/beta-catenin signaling. It has been proposed that DKKI alone or in combination with AFP is better than AFP alone for HCC diagnosis, especially for patients with normal AFP values or at early stage of HCC. . $4,74-76^{-1}$

GP73 This is a type II Golgi transmembrane protein. GP73 is expressed primarily in the bile duct of epithelial cells and rarely in the hepatocytes of the normal human liver. Its expression is significantly increased in liver diseases including HCC. Therefore, GP73 has been suggested as a potential HCC serum marker and also as a potentially useful marker of general liver disease progression. ${ }^{24,77}$

PIVKA-II PIVKA-II (also known as DCP) is an abnormal prothrombin protein that is increased in the sera of patients with HCC. Zakhary et al proved that PIVKA-II has the ability to discriminate between different histopathological grades of HCC (early and late stages of HCC); this confers PIVKA-Il's high sensitivity and specificity in comparison to $\alpha$ FP. ${ }^{24,78,79}$

SCCA-IGM Under physiological conditions, it is found in the spinous and granular layers of normal squamous epithelium. Recent findings have identified overexpression of SCCA variants (SCCA-I, SCCA-2, and SCCA-PD) in all surgically resected HCC specimens but no expression in the normal livers. Specifically, SCCA-I and SCCA-2 isoforms protect neoplastic cells from apoptosis induced by several stimuli; in vivo experiments have demonstrated that SCCA-I promotes tumor growth. ${ }^{80,81}$

AFU AFU is a liposomal enzyme present in all mammalian cells, blood, and body fluids, which is involved in the degradation of a variety of fructose-containing fucoglyco-conjugates. AFU-based detection is able to diagnose $85 \%$ of patients with $\mathrm{HCC} 6$ months before the detection by ultrasonography. Consequently, AFU has been proposed as a promising tumor marker in the diagnosis of $\mathrm{HCC} .^{74,82,83}$

GPC3 This is a member of the heparan sulfate proteoglycan family. Glypicans regulate the activity of several signaling molecules including Wnts. GPC3 can be detected in the serum as a secreted protein in a subset of patients with HCC, but it is undetectable in healthy individuals; patients with hepatitis and cirrhosis; or benign hepatic lesions. Therefore, GPC3 protein detection by immunohistochemistry in liver biopsies is currently being used in the clinic to confirm HCC diagnosis when the malignant nature of the lesion is difficult to establish. ${ }^{84,85}$

IGF-II IGF-II is a 67-amino-acid polypeptide growth factor that is mainly produced by liver cells and plays a crucial role in normal fetal growth. IGF-II may play an important role in the development of HCC neovascularization. It has been observed that free-circulating IGF-II levels are significantly higher in HCC patients than in those with chronic liver disease. ${ }^{86,87}$

OPN OPN expression is found in normal bones and kidneys. Serum OPN levels are correlated with hepatic inflammation and fibrosis in heavy alcohol drinkers, Circulating levels of OPN are elevated in patients with liver lesions associated with HCV and HBV infections. Although data suggest a better performance of plasma OPN in the diagnosis of HCC, the role of this biomarker still needs validation. ${ }^{88}$

miRNAs miRNAs are a class of small, noncoding, $\sim 22$ nucleotide-long RNAs, which may function as posttranscriptional regulators of gene expression. Many miRNAs are expressed in a tissue- or organ-specific manner, suggesting them as highly specific biomarkers. In the case of liver, miRNAs may enter the serum passively through apoptosis and necrosis or actively through secretion of exosomes and viral particles. ${ }^{89}$ Its detection in serum may provide a way to estimate miRNA activity in the liver. In addition to serum, miRNAs are detectable and remarkably stable in clinical samples such as blood, plasma, urine, and feces. Furthermore, frozen samples can be stored without substantial degradation because miRNAs are shown to be resistant to endogenous $\mathrm{RNase}$ activity, extreme $\mathrm{pH}$, high temperature, and multiple freeze-thaw cycles. ${ }^{90}$ In the last years, the expression profile of miRNAs in different types of samples such as HCC, cirrhotic patients, and healthy controls has been investigated. Some studies have shown the potential of the serum microRNAs miR-I9a, miR-296, miR-I30a, miR-195, miR-192, miR-34a, miR-I46a, miR-I5b, miR-2I, miR-I30b, miR-I83, miR-20a-5p, miR-320a, miR-324-3p, and miR-375 as early diagnostic HCC biomarkers. ${ }^{91-93}$

The new identification of panel of serum miRNAs (alone or combined with AFP) could be useful as early detection biomarkers for HCC screening with high accuracy in HCC diagnosis. It is important to mention that additional studies are required to confirm the clinical use of miRNAs in HCC diagnosis and prognosis. ${ }^{44,45}$

Exosomes $\quad$ Exosomes are cell-derived vesicles ranging from 30 to $100 \mathrm{~nm}$ that have been shown to affect gene expression in recipient cells. Exosomes contain characteristic RNA transcripts, including miRNAs, transfer RNAs, other types of noncoding RNAs, and several types of proteins depending on the cell of origin. Exosomes have gained great interest because they could serve as biomarkers for different types of cancer and could be easily detected in plasma. Thus, hepatocyte-derived exosomes may be also useful in HCC diagnosis. ${ }^{44-47}$

Abbreviations: AFP, alfa-fetoprotein; AFU, alfa-L-fucosidase; DCP, des- $\gamma$-carboxy-prothrombin; DKKI, dickkopf-I; GPC3; glypican 3; GP73, Golgi protein 73; HBV, hepatitis B virus; HCC, hepatocellular carcinoma; HCV, hepatitis C virus; IGF, insulin-like growth factor; miRNAs, microRNAs; OPN, osteopontin; PIVKA-II, protein induced by vitamin $\mathrm{K}$ absence or antagonist II; SCCA-IGM, serine protease inhibitor squamous cell carcinoma antigen-immunoglobulin M complex. 
Table 3 Therapies according to the HCC stage

\begin{tabular}{ll}
\hline HCC stage & Treatment \\
\hline Early & $\begin{array}{l}\text { Hepatic resection, liver transplantation, and ablation } \\
\text { by radiofrequency or percutaneous alcohol injection }\end{array}$ \\
Intermediate & $\begin{array}{l}\text { Chemoembolization } \\
\text { Advanced }\end{array}$ \\
& $\begin{array}{l}\text { Chemotherapy; the multikinase inhibitor sorafenib } \\
\text { is the most commonly used treatment option. }\end{array}$ \\
\hline
\end{tabular}

Note: Data on HCC stage. ${ }^{6,12,13,19}$

Abbreviation: HCC, hepatocellular carcinoma.

Table 4 Molecular targeted therapies for HCC

\begin{tabular}{|c|c|}
\hline$\overline{\text { Drug }}$ & Molecular mechanism \\
\hline \multicolumn{2}{|l|}{ First line } \\
\hline Sorafenib & $\begin{array}{l}\text { Multikinase tyrosine kinase inhibitor } \\
\text { (Raf, VEGFR, PDGFR) }\end{array}$ \\
\hline Sunitinib & $\begin{array}{l}\text { Multi-targeting receptor tyrosine kinase } \\
\text { inhibitor }{ }^{12,13,95,96}\end{array}$ \\
\hline Brivanib & $\begin{array}{l}\text { Multikinase tyrosine kinase inhibitor } \\
\text { (VEGFR, FGFR) })^{12,13,95}\end{array}$ \\
\hline Linifanib & $\begin{array}{l}\text { PDGFR/VEGFR tyrosine kinase } \\
\text { inhibitor }\end{array}$ \\
\hline Lenvatinib & $\begin{array}{l}\text { Multikinase tyrosine kinase inhibitor } \\
\text { (VEGFR2 and VEGFR3) }^{12}\end{array}$ \\
\hline \multicolumn{2}{|l|}{ Second line } \\
\hline Brivanib post-sorafenib & $\begin{array}{l}\text { Multikinase tyrosine kinase inhibitor } \\
\text { (VEGFR, PDGFR) }^{12,13,97}\end{array}$ \\
\hline Everolimus & mTOR inhibitor ${ }^{12,95}$ \\
\hline Ramucirumab & Monoclonal anti-VEGFR2 antibody ${ }^{12,95}$ \\
\hline Regorafenib & raf, VEGF, PDGF, Tie2 inhibitor ${ }^{\prime 2}$ \\
\hline Tivantinib & c-Met inhibitor ${ }^{12,13}$ \\
\hline Cabozantinib & c-Met inhibitor ${ }^{\prime 2}$ \\
\hline Refametinib & MEK inhibitor ${ }^{12}$ \\
\hline
\end{tabular}

Abbreviations: FGFR, fibroblast growth factor receptor; mTOR, mechanistic target of rapamycin; PDGFR, platelet-derived growth factor receptor; VEGFR; vascular endothelial growth factor receptor.

treatment was proposed to be used in other types of liver tumors including HCC. ${ }^{25}$

Due to the very poor prognosis of HCC and its late detection, it is essential to find early tumor markers as well as new therapeutic targets and potential anticancer drugs. Ion channels seem to be promising alternative tools in oncology to reach these goals.

\section{Eag I potassium channels as potential early biomarkers and alternative tools for HCC prevention and treatment}

Several reports have shown the abnormal expression of potassium channels in many tumors. Especially, the voltage-gated potassium channel ether à-go-go-1 (Eag1, KCNH1, Kv10.1) has gained enormous interest in cancer research because of its oncogenic properties. ${ }^{26-28} \mathrm{Eag} 1$ is overexpressed in most human tumors, including liver, cervical, lung, breast, colon, and prostate cancer. ${ }^{26-32}$ Eag1 channels have been also proposed as early tumor biomarkers and therapeutic targets for different types of cancers. ${ }^{30-36}$ In accordance with its role in cell proliferation, inhibition of Eag1 reduces tumor cell proliferation in vitro and in vivo. ${ }^{26,28,32,33,35,37-40}$ Astemizole is an antihistamine that has gained great interest as a potential anticancer drug because it targets several proteins involved in cancer, including Eag1 channels, histamine receptors, and proteins involved in drug resistance ${ }^{41}$ Cells expressing Eag1 and treated with astemizole display lower cell proliferation; it is hypothesized that this cell proliferation inhibition may be due to astemizole's blockage of the Eag1 channel. ${ }^{32,33,37,42}$ In vivo studies showed that astemizole administration reduced the growth rate of xenograft tumors in mice implanted with Eag1-expressing cells. ${ }^{42}$ Thus, Eag1 channels are inhibited by astemizole and may be potential targets for cancer therapy. ${ }^{26,27,32,41}$ Because Eag1 expression has been reported in human biopsies from liver tumors, ${ }^{30}$ astemizole may be used as an anticancer therapy in patients with liver cancer.

Considering the poor prognosis of HCC, our research group investigated if Eag1 channels could serve as early HCC markers by studying Eag1 expression during tumor development in vivo. We used the already established animal model of HCC development, where chronicle injection with diethylnitrosamine (DEN) induces liver injury in rats. This model recapitulates the sequential change from cirrhosis to $\mathrm{HCC}$, as it occurs in humans. Interestingly, we observed high Eag1 mRNA expression in most of the DEN-treated groups, and strong Eag1 protein expression was observed at early stages of HCC development ${ }^{32}$ (Figure 1). Eag1 expression at the mRNA and protein level was clearly higher in cirrhotic tissues and preneoplastic lesions, in comparison to normal livers. ${ }^{32}$ Thus, our results showed the potential of Eag 1 channels as HCC early biomarkers.

We also observed that astemizole clearly prevented the development of HCC; the antihistamine prevented tumor formation when administered either from the start of DEN treatment or from week 12 of DEN treatment, when cirrhosis is already present. ${ }^{32}$ These results suggest that astemizole might be used as a chemopreventive agent in patients at risk of developing liver cancer. Additionally, astemizole could be used as an antineoplastic because when administered at the end of the DEN treatment, the animals did not develop big tumors as those receiving astemizole, ${ }^{32}$ suggesting that this compound may induce tumor regression.

Eag1 mRNA and protein are expressed in the human HCC cell lines HepG2 and HuH7; accordingly, astemizole 
decreased their cell proliferation and induced apoptosis. ${ }^{32}$ Taken together, all these results suggest that astemizole may be a novel therapeutic approach for HCC patients.

Other types of channels and transporters also displayed a differential expression during HCC development, suggesting other channels as potential early markers and/or therapeutic targets of HCC. ${ }^{43}$

\section{Future research on Eag I channels as HCC biomarkers and challenges}

The discovery of cancer biomarkers in recent years has become a major focus of cancer research. The development of new technologies, especially genomics and proteomics, has made possible the identification and discovery of potential biomarkers. In the recent years, the study of microRNAs and exosomes has gained great interest to diagnose HCC in at-risk patients. ${ }^{44-48} \mathrm{An}$ ideal tumor marker would be DNA-, RNA-, protein-, or antibody-based measurable in serum, urine, or sputum. ${ }^{49,50}$

Because Eag1 may have clinical potential as an early biomarker for $\mathrm{HCC},{ }^{32}$ it would be very interesting to track Eag1 protein expression using sensitive imaging techniques in human livers. Actually, when Eag1-expressing cells were injected into mice, the channel was detected in nonpalpable tumors ${ }^{51}$ using dye-tagged antibodies and imaging techniques. This type of approach might also be used to screen Eag1 expression in the liver of patients at risk of developing HCC and in HCC animal models. It would be also very important to study exosomes containing Eag1 at different stages of $\mathrm{HCC}$ in liquid biopsies. Astemizole may be a very promising preventive option for patients at risk of developing $\mathrm{HCC}$ as well as a hopeful therapeutic option for HCC patients. ${ }^{32}$ Therefore, clinical trials testing the effect of astemizole alone or in combination with other antineoplastic drugs in patients either at risk of developing or with HCC should be performed. In addition, detection of Eag 1 could be included in the surveillance scheme for patients who are at risk of developing HCC for timely diagnosis (Figure 2).

The precise role of Eag1 channels in HCC development remains to be elucidated. This might be accomplished in different manners, such as the use of mouse models like Eag1 knockout mice. ${ }^{52}$ It would be very interesting to investigate whether these knockout mice are resistant to the development of liver cirrhosis and HCC. ${ }^{32}$

Another very important issue to investigate is the mechanism of the oncogenic potential of the channel. Eag1 expression is regulated by the p53-mir-34-E2F1pathway. ${ }^{53}$ It has been suggested that mutated or inactive p53 increases Eag1 expression; p53 is mutated in most tumors including HCC. ${ }^{54}$ The increased Eag1 expression could lead to the activation of different proteins such as HIF-1, cyclins D/E, ERK1/2, and FAK complex as well as increase the intracellular calcium concentration and induce vascular endothelial growth factor release. All these changes may lead to angiogenesis, therapy resistance, cell proliferation, migration, and survival, contributing to carcinogenesis. ${ }^{55}$ However, the precise molecular mechanism of how Eag1 may contribute to HCC development remains to be elucidated.

Liquid biopsies studying $\mathrm{HCC}$ circulating cells may provide novel insights into HCC diagnosis and prognosis. ${ }^{56,57}$ Because Eag1 channels might serve as early markers for different types of cancer, it may be very useful to detect Eag1 mRNA/protein expression in such liquid biopsies. This approach may help to find the urgently needed HCC markers, which are hardly available. However, one of the biggest challenges to overcome would be the sensitivity and specificity of the HCC detection method based on Eag1 expression. In addition, prospective studies should be made to know if cirrhotic patients expressing Eag1 channels would develop HCC.

\section{Conclusion}

Because of the poor prognosis of $\mathrm{HCC}$, it is very important to find novel preventive and therapeutic alternatives. Recent studies have suggested that astemizole may be a very promising preventive option for patients at risk of developing $\mathrm{HCC}$ as well as a hopeful therapeutic option for HCC patients. It has also been recently proposed that Eag1 may be a potential early biomarker of HCC. Astemizole-based prevention and therapy and early Eag1 detection in the liver should help to reduce mortality from this disease.

\section{Disclosure}

The authors report no conflicts of interest in this work.

\section{References}

1. Mittal S, El-Serag HB. Epidemiology of hepatocellular carcinoma: consider the population. J Clin Gastroenterol. 2013;47 Supp1:S2-S6.

2. Jemal A, Siegel R, Ward E, et al. Cancer statistics, 2008. CA Cancer J Clin. 2008;58(2):71-96.

3. Chuang SC, La Vecchia C, Boffetta P. Liver cancer: descriptive epidemiology and risk factors other than HBV and HCV infection. Cancer Lett. 2009;286(1):9-14.

4. Venook AP, Papandreou C, Furuse J, de Guevara LL. The incidence and epidemiology of hepatocellular carcinoma: a global and regional perspective. Oncologist. 2010;15(Suppl 4):5-13.

5. Herbst DA, Reddy KR. Risk factors for hepatocellular carcinoma. Clin Liver Dis. 2012;1(6):180-182. 
6. Sherman M, Llovet JM. Smoking, hepatitis B virus infection, and development of hepatocellular carcinoma. J Natl Cancer Inst. 2011;103(22):1642-1643.

7. Bruix J, Llovet JM. Hepatitis B virus and hepatocellular carcinoma. J Hepatol. 2003;39(Suppl 1):S59-S63.

8. Gao J, Xie L, Yang WS, et al. Risk factors of hepatocellular carcinoma - current status and perspectives. Asian Pac J Cancer Prev. 2012;13(3):743-752.

9. Hong M, Li S, Tan HY, Wang N, Tsao SW, Feng Y. Current status of herbal medicines in chronic liver disease therapy: the biological effects, molecular targets and future prospects. Int $J$ Mol Sci. 2015;16(12):28705-28745.

10. Farazi PA, DePinho RA. Hepatocellular carcinoma pathogenesis: from genes to environment. Nat Rev Cancer. 2006;6(9):674-687.

11. Imawari M. Liver cancer, prevention and early diagnosis. JMAJ. 2002;5(3):130-133.

12. Wang CH, Wey KC, Mo LR, Chang KK, Lin RC, Kuo JJ. Current trends and recent advances in diagnosis, therapy, and prevention of hepatocellular carcinoma. Asian Pac J Cancer Prev. 2015;16(9):3595-3604.

13. Ingle PV, Samsudin SZ, Chan PQ, et al. Development and novel therapeutics in hepatocellular carcinoma: a review. Ther Clin Risk Manag. 2016;12:445-455.

14. Giacomin A, Cazzagon N, Sergio A, Vanin V, Farinati F. Hepatitis B virus-related hepatocellular carcinoma: primary, secondary, and tertiary prevention. Eur J Cancer Prev. 2011;20(5):381-388.

15. Chan CY, Lee SD, Lo KJ. Legend of hepatitis B vaccination: the Taiwan experience. J Gastroenterol Hepatol. 2004;19(2):121-126.

16. Dhanasekaran R, Limaye A, Cabrera R. Hepatocellular carcinoma: current trends in worldwide epidemiology, risk factors, diagnosis, and therapeutics. Hepat Med. 2012;4:19-37.

17. Chan HL, Wong GL, Tse CH, Chan HY, Wong VW. Viral determinants of hepatitis B surface antigen seroclearance in hepatitis B e antigen-negative chronic hepatitis B patients. J Infect Dis. 2011;204(3): 408-414.

18. Llovet JM, Bruix J. Novel advancements in the management of hepatocellular carcinoma in 2008. J Hepatol. 2008;48 suppl 1:S20-S37.

19. Befeler AS, Di Bisceglie AM. Hepatocellular carcinoma: diagnosis and treatment. Gastroenterology. 2002;122(6):1609-1619.

20. Johnson PJ. The role of serum alpha-fetoprotein estimation in the diagnosis and management of hepatocellular carcinoma. Clin Liver Dis. 2001;5(1):145-159.

21. Parikh NI, Vasan RS. Assessing the clinical utility of biomarkers in medicine. Biomark Med. 2007;1(3):419-436.

22. Hartwell L, Mankoff D, Paulovich A, Ramsey S, Swisher E. Cancer biomarkers: a systems approach. Nat Biotechnol. 2006;24(8): 905-908.

23. Gonzalez SA. Novel biomarkers for hepatocellular carcinoma surveillance: has the future arrived? Hepatobiliary surgery and nutrition. 2014;3(6):410-414.

24. Zhu K, Dai Z, Zhou J. Biomarkers for hepatocellular carcinoma: progression in early diagnosis, prognosis, and personalized therapy. Biomark Res. 2013;1(1):1-8.

25. Edhemovic I, Brecelj E, Gasljevic G, et al. Intraoperative electrochemotherapy of colorectal liver metastases. J Surg Ocol. 2014;110(3): 320-327.

26. Pardo LA, Stühmer W. Eag1: an emerging oncological target. Cancer Res. 2008;68(6):1611-1613.

27. Wulff H, Castle NA, Pardo LA. Voltage-gated potassium channels as therapeutic targets. Nat Rev Drug Discov. 2009;8(12):982-1001.

28. Pardo LA, del Camino D, Sanchez A, et al. Oncogenic potential of EAG $\mathrm{K}(+)$ channels. EMBO J. 1999;18(20):5540-5547.

29. Rodríguez-Rasgado JA, Acuña-Macías I, Camacho J. Eag1 channels as potential cancer biomarkers. Sensors (Basel). 2012;12(5):5986-5995.

30. Hemmerlein B, Weseloh RM, Mello de Queiroz F, et al. Overexpression of Eag1 potassium channels in clinical tumours. Mol Cancer. 2006;5:41.

31. Ousingsawat J, Spitzner M, Puntheeranurak S, et al. Expression of voltage-gated potassium channels in human and mouse colonic carcinoma. Clin Cancer Res. 2007;13(3):824-831.
32. de Guadalupe Chávez-López M, Pérez-Carreón JI, Zuñiga-García V, et al. Astemizole-based anticancer therapy for hepatocellular carcinoma (HCC), and Eag1 channels as potential early-stage markers of HCC. Tumor Biol. 2015;36(8):6149-6158.

33. Diaz L, Ceja-Ochoa I, Restrepo-Angulo I, et al. Estrogens and human papilloma virus oncogenes regulate human ether-a-go-go-1 potassium channel expression. Cancer Res. 2009;69(8):3300-3307.

34. Farias LM, Ocana DB, Diaz L, et al. Ether a go-go potassium channels as human cervical cancer markers. Cancer Res. 2004;64(19):6996-7001.

35. Gómez-Varela D, Zwick-Wallasch E, Knötgen H, et al. Monoclonal antibody blockade of the human Eag1 potassium channel function exerts antitumor activity. Cancer Res. 2007;67(15):7343-7349.

36. Ortiz CS, Montante-Montes D, Saqui-Salces M, et al. Eag1 potassium channels as markers of cervical dysplasia. Oncol Rep. 2011;26(6):1377-1383.

37. Ouadid-Ahidouch H, Le Bourhis X, Roudbaraki M, Toillon RA, Delcourt P, Prevarskaya N. Changes in the K+ current-density of MCF-7 cells during progression through the cell cycle: possible involvement of a h-ether.a-gogo K+ channel. Receptors Channels. 2001;7(5):345-356.

38. Weber C, Mello de Queiroz F, Downie BR, Suckow A, Stuhmer W, Pardo LA. Silencing the activity and proliferative properties of the human EagI potassium channel by RNA Interference. $J$ Biol Chem. 2006;281(19):13030-13037.

39. Garcia-Becerra R, Diaz L, Camacho J, et al. Calcitriol inhibits ether-a go-go potassium channel expression and cell proliferation in human breast cancer cells. Exp Cell Res. 2010;316(3):433-442.

40. Gavrilova-Ruch O, Schonherr K, Gessner G, et al. Effects of imipramine on ion channels and proliferation of IGR1 melanoma cells. J Membr Biol. 2002;188(2):137-149.

41. Garcia-Quiroz J, Camacho J. Astemizole: an old anti-histamine as a new promising anti-cancer drug. Anticancer Agents Med Chem. 2011;11(3): 307-314.

42. Downie BR, Sanchez A, Knötgen H, et al. Eag1 expression interferes with hypoxia homeostasis and induces angiogenesis in tumors. $J$ Biol Chem. 2008;283(52):36234-36240.

43. Zúñiga-García V, Chávez-López Mde G, Quintanar-Jurado V, et al. Differential expression of ion channels and transporters during hepatocellular carcinoma development. Dig Dis Sci. 2015;60(8):2373-2383.

44. Hayes CN, Chayama K. MicroRNAs as biomarkers for liver disease and hepatocellular carcinoma. Int J Mol Sci. 2016;17(3):280.

45. Lin XJ, Chong Y, Guo ZW, et al. A serum microRNA classifier for early detection of hepatocellular carcinoma: a multicentre, retrospective, longitudinal biomarker identification study with a nested case-control study. Lancet Oncol. 2015;16(7):804-815.

46. Kaiser J. Malignant messengers. Science. 2016;352(6282):164-166.

47. Minciacchi VR, Freeman MR, Di Vizio D. Extracellular vesicles in cancer: exosomes, microvesicles and the emerging role of large oncosomes. Semin Cell Dev Biol. 2015;40:41-51.

48. Feo F, Pascale RM. Multifocal hepatocellular carcinoma: intrahepatic metastasis or multicentric carcinogenesis? Ann Transl Med. 2015;3(1):4.

49. Verma M, Kagan J, Sidransky D, Srivastava S. Proteomic analysis of cancer-cell mitochondria. Nat Rev Cancer. 2003;3(10):789-795.

50. Kumar S, Mohan A, Guleria R. Biomarkers in cancer screening, research and detection: present and future: a review. Biomarkers. 2006;11(5):385-405.

51. Pardo LA, Contreras-Jurado C, Zientkowska M, Alves F, Stühmer W. Role of voltage-gated potassium channels in cancer. J Membr Biol. 2005;205(3):115-124.

52. Ufartes R, Schneider T, Mortensen LS, et al. Behavioural and functional characterization of Kv10.1 (Eag1) knockout mice. Hum Mol Genet. 2013; 22(11):2247-2262.

53. Lin $\mathrm{H}$, Li Z, Chen $\mathrm{C}$, et al. Transcriptional and post-transcriptional mechanisms for oncogenic overexpression of ether a go-go $\mathrm{K}+$ channel. PLoS One. 2011;6(5):e20362.

54. De Benedetti VM, Welsh JA, Yu MC, Bennett WP. p53 mutations in hepatocellular carcinoma related to oral contraceptive use. Carcinogenesis. 1996;17(1):145-149.

55. Ouadid-Ahidouch H, Ahidouch A, Pardo LA. Kv10.1 K(+) channel: from physiology to cancer. Pflugers Arch. 2016;468(5):751-762. 
56. Xue R, Li R, Guo H, et al. Variable intra-tumor genomic heterogeneity of multiple lesions in patients with hepatocellular carcinoma. Gastroenterology. 2016;150(4):998-1008.

57. Zaki MYW, Reeves HL. The genetic heterogeneity of hepatocellular carcinoma and the implications for personalised medicine. Transl Cancer Res. 2016;5(S1):S1-S4.

58. Starr SP, Raines D. Cirrhosis: diagnosis, management, and prevention. Am Family Physician. 2011;84(12):1353-1359.

59. Tsochatzis EA, Bosch J, Burroughs AK. Liver cirrhosis. Lancet. 2014;383(9930):1749-1761.

60. Kew MC. Hepatitis B virus x protein in the pathogenesis of hepatitis B virus-induced hepatocellular carcinoma. J Gastroenterol Hepatol. 2011;26 suppl 1:144-152.

61. Geng M, Xin X, Bi LQ, Zhou LT, Liu XH. Molecular mechanism of hepatitis B virus $\mathrm{X}$ protein function in hepatocarcinogenesis. World $J$ Gastroenterol. 2015;21(38):10732-10738.

62. Tarocchi M, Polvani S, Marroncini G, Galli A. Molecular mechanism of hepatitis B virus-induced hepatocarcinogenesis. World J Gastroenterol. 2014;20(33):11630-11640.

63. Wirth TC, Manns MP. The impact of the revolution in hepatitis C treatment on hepatocellular carcinoma. Ann Oncol. 2016;27(8):1467-1474.

64. Jahan S, Ashfaq UA, Qasim M, Khaliq S, Saleem MJ, Afzal N. Hepatitis C virus to hepatocellular carcinoma. Infect Agent Cancer. 2012;7(1):2.

65. Kew MC. Aflatoxins as a cause of hepatocellular carcinoma. J Gastrointestin Liver Dis. Sep 2013;22(3):305-310.

66. Testino G, Leone S, Borro P. Alcohol and hepatocellular carcinoma: a review and a point of view. World J Gastroenterol. 2014;20(43): 15943-15954.

67. Ali Kamkar MM, Ahmad R, Alsmadi O, Behbehani K. Insight into the impact of diabetes mellitus on the increased risk of hepatocellular carcinoma: mini-review. J Diabet Metab Disord. 2014;13:57.

68. Streba LA, Vere CC, Rogoveanu I, Streba CT. Nonalcoholic fatty liver disease, metabolic risk factors, and hepatocellular carcinoma: an open question. World J Gastroenterol. 2015;21(14):4103-4110.

69. Kikuchi L, Oliveira CP, Carrilho FJ. Nonalcoholic fatty liver disease and hepatocellular carcinoma. Biomed Res Int. 2014;2014:6.

70. Kew MC. Hepatic iron overload and hepatocellular carcinoma. Liver Cancer. 2014;3(1):31-40.

71. Purohit V, Rapaka R, Kwon OS, Song BJ. Roles of alcohol and tobacco exposure in the development of hepatocellular carcinoma. Life Sci. 2013;92(1):3-9.

72. Kew MC. Obesity as a cause of hepatocellular carcinoma. Ann Hepatol. 2015;14(3):299-303.

73. Arrieta O, Cacho B, Morales-Espinosa D, Ruelas-Villavicencio A, Flores-Estrada D, Hernandez-Pedro N. The progressive elevation of alpha fetoprotein for the diagnosis of hepatocellular carcinoma in patients with liver cirrhosis. BMC Cancer. 2007;7:28.

74. Prieto PA, Cha CH. DKK1 as a serum biomarker for hepatocellular carcinoma. Hepatobiliary Surge Nutr. 2012;2(3):127-128.

75. Tung EK, $\mathrm{Ng} \mathrm{IO}$. Significance of serum DKK1 as a diagnostic biomarker in hepatocellular carcinoma. Future Oncol. 2012;8(12):1525-1528.

76. Shen Q, Fan J, Yang XR, et al. Serum DKK1 as a protein biomarker for the diagnosis of hepatocellular carcinoma: a large-scale, multicentre study. Lancet Oncol. 2012;13(8):817-826.

77. Xu Z, Liu L, Pan X, et al. Serum Golgi protein 73 (GP73) is a diagnostic and prognostic marker of chronic HBV liver disease. Medicine (Baltimore). 2015;94(12):e659.

78. Zakhary NI, Khodeer SM, Shafik HE, Abdel Malak CA. Impact of PIVKA-II in diagnosis of hepatocellular carcinoma. JAdv Res. 2013;4(6): 539-546.
79. Yu R, Ding S, Tan W, et al. Performance of protein induced by vitamin $\mathrm{k}$ absence or antagonist-II (PIVKA-II) for hepatocellular carcinoma screening in Chinese population. Hepat Mon. 2015;15(7):e28806.

80. Beneduce L, Castaldi F, Marino M, et al. Squamous cell carcinoma antigen-immunoglobulin $\mathrm{M}$ complexes as novel biomarkers for hepatocellular carcinoma. Cancer. 2005;103(12):2558-2565.

81. Schütte K, Schulz C, Link A, Malfertheiner P. Current biomarkers for hepatocellular carcinoma: Surveillance, diagnosis and prediction of prognosis. World J Hepatol. 2015;7(2):139-149.

82. Wang K, Guo W, Li N, et al. Alpha-1-fucosidase as a prognostic indicator for hepatocellular carcinoma following hepatectomy: a large-scale, long-term study. Br J Cancer. 2014;110(7):1811-1819.

83. Zhang SY, Lin BD, Li BR. Evaluation of the diagnostic value of alpha1-fucosidase, alpha-fetoprotein and thymidine kinase 1 with ROC and logistic regression for hepatocellular carcinoma. FEBS Open Bio. 2015;5:240-244.

84. Filmus J, Capurro M. Glypican-3: a marker and a therapeutic target in hepatocellular carcinoma. FEBS J. 2013;280(10):2471-2476.

85. Wang HL, Anatelli F, Zhai QJ, Adley B, Chuang ST, Yang XJ. Glypican-3 as a useful diagnostic marker that distinguishes hepatocellular carcinoma from benign hepatocellular mass lesions. Arch Pathol Lab Med. 2008;132(11):1723-1728.

86. Wang Z, Ruan YB, Guan Y, Liu SH. Expression of IGF-II in early experimental hepatocellular carcinomas and its significance in early diagnosis. World J Gastroenterol. 2003;9(2):267-270.

87. Wang L, Yao M, Dong Z, Zhang Y, Yao D. Circulating specific biomarkers in diagnosis of hepatocellular carcinoma and its metastasis monitoring. Tumour Biol. 2014;35(1):9-20.

88. Simão A, Madaleno J, Silva N, et al. Plasma osteopontin is a biomarker for the severity of alcoholic liver cirrhosis, not for hepatocellular carcinoma screening. BMC Gastroenterol. 2015;15:73.

89. Novellino L, Rossi RL, Bonino F, et al. Circulating hepatitis B surface antigen particles carry hepatocellular microRNAs. PLoS One. 2012;7(3):e31952

90. Jiang L, Cheng Q, Zhang BH, Zhang MZ. Circulating microRNAs as biomarkers in hepatocellular carcinoma screening: a validation set from China. Medicine (Baltimore). 2015;94(10):e603.

91. Liu AM, Yao TJ, Wang W, et al. Circulating miR-15b and miR-130b in serum as potential markers for detecting hepatocellular carcinoma: a retrospective cohort study. BMJ Open. 2012;2(2):e000825.

92. Motawi TK, Shaker OG, El-Maraghy SA, Senousy MA. Serum microRNAs as potential biomarkers for early diagnosis of hepatitis $\mathrm{C}$ virus-related hepatocellular carcinoma in Egyptian patients. PLoS One. 2015;10(9):e0137706.

93. Wen Y, Han J, Chen J, et al. Plasma miRNAs as early biomarkers for detecting hepatocellular carcinoma. Int J Cancer. 2015;137(7): 1679-1690.

94. Kudo M, Imanaka K, Chida N, et al. Phase III study of sorafenib after transarterial chemoembolisation in Japanese and Korean patients with unresectable hepatocellular carcinoma. Eur J Cancer. 2011;47(14):2117-2127.

95. European Association for Study of Liver; European Organisation for Research and Treatment. EASL-EORTC clinical practice guidelines: management of hepatocellular carcinoma. Eur J Cancer. 2012;48(5):599-641.

96. Cheng AL, Kang YK, Lin DY, et al. Sunitinib versus sorafenib in advanced hepatocellular cancer: results of a randomized phase III trial. J Clin Oncol. 2013;31(32):4067-4075.

97. Kudo M, Han G, Finn RS, et al. Brivanib as adjuvant therapy to transarterial chemoembolization in patients with hepatocellular carcinoma: a randomized phase III trial. Hepatology. 2014;60(5):1697-1707. 


\section{Publish your work in this journal}

Biologics: Targets and Therapy is an international, peer-reviewed journal focusing on the patho-physiological rationale for and clinical application of Biologic agents in the management of autoimmune diseases, cancers or other pathologies where a molecular target can be identified. This journal is indexed on PubMed Central, EMBase, and Scopus.
The manuscript management system is completely online and includes a very quick and fair peer-review system, which is all easy to use Visit http://www.dovepress.com/testimonials.php to read real quotes from published authors.

Submit your manuscript here: https://www.dovepress.com/biologics-targets-and-therapy-journal 